\title{
Radiative symmetry breaking from interacting UV fixed points
}

\author{
Steven Abel ${ }^{1,2, *}$ and Francesco Sannino ${ }^{2,3, \dagger}$ \\ ${ }^{1}$ IPPP, Durham University, South Road, Durham DHI 3LE, United Kingdom \\ ${ }^{2}$ Theory Division, Cern, Route de Meyrin, 1211 Geneva 23, Switzerland \\ ${ }^{3} \mathrm{CP}^{3}$-Origins \& the Danish Institute for Advanced Study, University of Southern Denmark, \\ Campusvej 55, DK-5230 Odense, Denmark \\ (Received 25 April 2017; published 28 September 2017)
}

\begin{abstract}
It is shown that the addition of positive mass-squared terms to asymptotically safe gauge-Yukawa theories with perturbative UV fixed points leads to calculable radiative symmetry breaking in the IR. This phenomenon, and the multiplicative running of the operators that lies behind it, is akin to the radiative symmetry breaking that occurs in the supersymmetric standard model.
\end{abstract}

DOI: $10.1103 /$ PhysRevD.96.056028

\section{INTRODUCTION}

It is interesting to search for theories with interacting fixed points that do not possess internal hierarchies, as they may be useful starting points to develop an asymptotically safe standard model. The purpose of this paper is to examine in this context the perturbative example of such a theory presented in Ref. [1], and developed in Refs. [2-5].

Our central point will be that these theories allow radiative symmetry breaking driven by arbitrary masssquared operators. The couplings (i.e., the ensemble of masses-squared) run multiplicatively from the UV fixed point. In this sense their renormalization group (RG) behavior is similar to, and as controllable as, that of the soft-terms in supersymmetry (SUSY). Moreover we find that, as in the minimal supersymmetric standard model (MSSM), a positive mass-squared operator in the UV induces negative mass-squared operators in the IR due to large couplings.

The end result is a calculable radiatively induced symmetry breaking, exactly analogous to that in the MSSM [6], that is proportional to the explicit degree of flavor breaking added to the mass-squared terms. It is a very different and more complete paradigm for radiative breaking than the one normally invoked in the context of scale invariance, namely the Coleman-Weinberg $(\mathrm{CW})$ mechanism [7-47]. The latter sets the masses to zero at the origin of field space, with some hopeful words that this could well be a prediction of scale invariance. Here we emphasize that UV scale invariance does not prefer any value for mass-terms, since they are relevant operators, and the fixed point is completely blind to them. Therefore one

\footnotetext{
s.a.abel@durham.ac.uk

sannino@cp3-origins.net
}

Published by the American Physical Society under the terms of the Creative Commons Attribution 4.0 International license. Further distribution of this work must maintain attribution to the author(s) and the published article's title, journal citation, and DOI. may perfectly consistently choose a mass-squared parameter to be "small" (relative to the dimensional transmutation scale, say) in which case the $\mathrm{CW}$ version of radiative symmetry breaking can in principle still operate (although not as it turns out perturbatively in the case we discuss), or one may choose it to be large. Either possibility is consistent with exact quantum UV scale invariance since the "starting values" of the dimensionful (i.e. relevant) parameters at some RG scale are free parameters, again much like the quark masses in QCD. Furthermore both cases should be thought of as radiative symmetry breaking, just driven by different operators. This picture is entirely different from flows governed by IR fixed points, in which relevant operators do determine the fixed point.

We should explain why an interacting fixed point increases the calculability and predictivity. Suppose for example that one wished to compute perturbative corrections to the dimensionless couplings of the effective theory. Such corrections would have a UV "divergence" going as $1 / \gamma$ where $\gamma$ is the anomalous dimension of the operator at the fixed point. If the fixed point is Gaussian then the anomalous dimensions at the fixed point are zero and this corresponds to a real divergence which tells us that generically the couplings are simply given by their settings at renormalization time $-t=\log \left(\mu_{0} / \mu\right)=0$.

By contrast, if the fixed point is interacting then anomalous dimensions at the fixed point are nonzero, and radiative corrections will simply be finite terms going as $1 / \gamma$. In particular they are insensitive to $\mu_{0}$ which we may as well take to be infinite. (These points were discussed in some detail in Ref. [48].) If the fixed point is interacting and strongly coupled, a perturbative treatment is impossible, but nevertheless nonzero anomalous dimensions make the corresponding couplings insensitive to the precise details of the approach to the UV fixed point, thereby restoring predictivity. Of course the anomalous dimensions may not be large enough to regulate classically relevant operators: as mentioned above, such operators simply experience multiplicative RG running in the usual way from values 
chosen at some initial scale $\mu_{0}$ (much like quark masses), but cannot disrupt the UV fixed point, so the asympotic safety of the setup is immune to them.

The asymptotically safe theories of Ref. [1] that we will be using here lie somewhere between these two extremes. By choosing a theory with a weakly interacting UV fixed point we recover the benefits of predictivity and control over the effective potential, but at the same time keep the theory under good perturbative control. This optimization is reminiscent of the Banks-Zaks IR fixed point [49], which can be made arbitrarily weakly interacting and hence perturbatively tractable, in a particular (Veneziano) largecolor/large-flavor limit.

Of course this work follows on from a large body of literature that has discussed asymptotic safety and more generally the consequences of UV scale invariance both with and without gravity: [48,50-59]). (For a review see [60]). The object of this paper is to place radiative symmetry breaking in such frameworks on the same footing as it is in the MSSM.

\section{THE THEORY, UV FIXED POINT, AND CRITICAL CURVE}

We begin by describing the behavior of the weakly interacting gauge-Yukawa theories that we will be using, and in particular their phase diagrams and RG flow. Consider a theory with $S U\left(N_{C}\right)$ gauge fields $A_{\mu}^{a}$ and field strength $F_{\mu \nu}^{a}\left(a=1, \ldots, N_{C}\right), N_{F}$ flavors of fermions $Q_{i}$ $\left(i=1, \ldots, N_{F}\right)$ in the fundamental representation, and an $N_{F} \times N_{F}$ complex matrix scalar field $H$ uncharged under the gauge group. At the fundamental level the Lagrangian is given by the sum of the Yang-Mills term, the fermion and scalar kinetic terms, the Yukawa interaction, and scalar self-interaction terms,

$$
\begin{aligned}
L= & -\frac{1}{2} \operatorname{Tr} F^{\mu \nu} F_{\mu \nu}+\operatorname{Tr}\left(\bar{Q}_{i D Q}\right)+\operatorname{Tr}\left(\partial_{\mu} H^{\dagger} \partial^{\mu} H\right) \\
& +y \operatorname{Tr}\left(\bar{Q}_{L} H Q_{R}+\bar{Q}_{R} H^{\dagger} Q_{L}\right)-u \operatorname{Tr}\left(H^{\dagger} H\right)^{2} \\
& -v\left(\operatorname{Tr} H^{\dagger} H\right)^{2},
\end{aligned}
$$

where the decomposition $Q=Q_{L}+Q_{R}$ with $Q_{L / R}=$ $\frac{1}{2}\left(1 \pm \gamma_{5}\right) Q$ is understood. The trace $\mathrm{Tr}$ indicates the trace over both color and flavor indices.

The model has four coupling constants given by the gauge coupling, the Yukawa coupling $y$, and the quartic scalar couplings $u$ and the double-trace scalar coupling $v$ :

$\alpha_{g}=\frac{g^{2} N_{C}}{(4 \pi)^{2}}, \quad \alpha_{y}=\frac{y^{2} N_{C}}{(4 \pi)^{2}}, \quad \alpha_{h}=\frac{u N_{F}}{(4 \pi)^{2}}, \quad \alpha_{v} v=\frac{v N_{F}^{2}}{(4 \pi)^{2}}$.

We have already rescaled the coupling constants by the appropriate powers of $N_{C}$ and $N_{F}$ to work in the Veneziano limit. When necessary we will use a shorthand notation $\alpha_{i}$ with $i=(g, y, h, v)$. As mentioned in the Introduction we will be considering the large color and large flavor Veneziano limit, in order to have an interacting fixed point which is nevertheless arbitrarily weakly coupled. Therefore it is convenient to introduce a control parameter which in the Veneziano limit is a continuous and arbitrarily small constant

$$
\epsilon=\frac{N_{F}}{N_{C}}-\frac{11}{2} .
$$

Asymptotic freedom is lost for positive values of $\epsilon$.

Reference [1] discovered a number of fixed points for this model. However there is one fixed point that is unique in that it has only one relevant direction with the other three being irrelevant. Since every relevant direction loses predictivity (as it is formally zero at the fixed point and must be set by hand) this fixed point is of great interest. To the maximum currently achievable order in perturbation theory and properly respecting the Weyl consistency conditions it is obtained for

$$
\begin{aligned}
& \alpha_{g}^{*}=0.4561 \epsilon+0.7808 \epsilon^{2}+\mathcal{O}\left(\epsilon^{3}\right) \\
& \alpha_{y}^{*}=0.2105 \epsilon+0.5082 \epsilon^{2}+\mathcal{O}\left(\epsilon^{3}\right) \\
& \alpha_{h}^{*}=0.1998 \epsilon+0.5042 \epsilon^{2}+\mathcal{O}\left(\epsilon^{3}\right),
\end{aligned}
$$

with the leading coefficients of $\epsilon$ corresponding to $\alpha_{g}^{*}=\frac{26}{57} \epsilon+\cdots, \quad \alpha_{y}^{*}=\frac{4}{19} \epsilon+\cdots \quad$ and $\quad \alpha_{h}^{*}=\frac{\sqrt{23}-1}{19} \epsilon+\cdots$ respectively. Note that the quartic scalar self-coupling is essential for this fixed point to exist. The remaining doubletrace scalar coupling $v$ has two possible fixed points, one of which is more perturbatively reliable and adds an irrelevant scaling direction to the theory, found to be at

$$
\begin{aligned}
\alpha_{v 1}^{*}= & \frac{-6 \sqrt{23+4 \epsilon}+3 \sqrt{4 \epsilon+6 \sqrt{23+4 \epsilon}+20}}{4 \epsilon+26} \alpha_{g}^{*} \\
& +\mathcal{O}\left(\alpha_{g}^{* 2}\right) .
\end{aligned}
$$

Numerically $\alpha_{v 1}^{*}=-0.1373 \epsilon$ up to quadratic corrections in $\epsilon$.

Following [1] by performing the perturbative expansion to high orders in $\epsilon$ one finds its radius of convergence to be

$$
\epsilon \leq \epsilon_{\max }=0.117 \cdots
$$

At $\epsilon_{\max }$ in (6), the NNLO equations display a bifurcation and the UV fixed point ceases to exist through a merger with a nonperturbative IR fixed point, and the relevant eigenvalue disappears at $\epsilon_{\max }$. The merger at $\epsilon_{\max }$ indicates that our working perturbative assumption $(\epsilon \ll 1)$ should be replaced by $0<\epsilon<\epsilon_{\max }$.

In the presence of more than one relevant direction the flow from the UV would be expected to emanate from a 


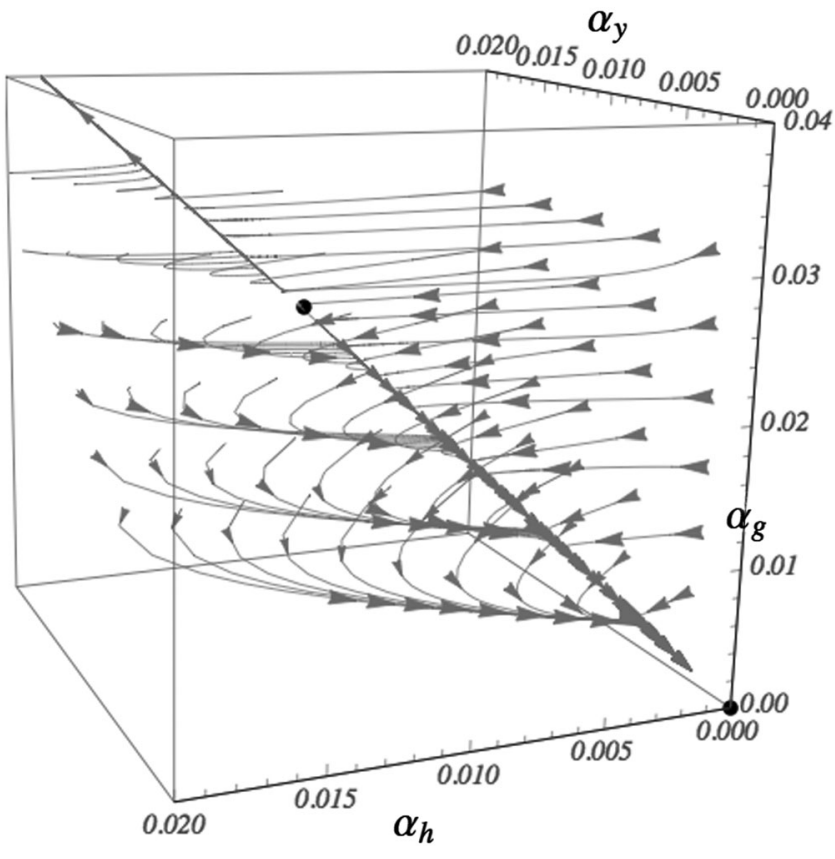

FIG. 1. The renormalization group flow of the marginal couplings from the UV fixed point and around the critical curve, towards the Gaussian IR fixed point.

critical surface, however with only one relevant direction the flow is along the critical curve shown in Fig. 1 toward the IR stable Gaussian fixed point in the infrared, and is therefore completely determined in terms of a single parameter which could be taken to be the gauge coupling itself. The arrows in the figure are at equal separation in renormalization "time", so it is clear that the flow to the critical curve happens much more rapidly than flow along it. In fact as discussed in Ref. [1] the relative rate of flow is proportional to $\epsilon$. Of course for the present discussion the flow emanates precisely from the UV fixed point of Eq. (4) marked in black, along the critical curve towards the Gaussian IR fixed point.

In scalar field theories we must also determine if the potential is stable. Ignoring the possible presence of relevant operators for the moment, we see that this is indeed the case at leading order since $\alpha_{h}^{*}+\alpha_{v 1}^{*}>0$, and it also the case for loop corrections as well $[1,3]$. Therefore there is no Coleman-Weinberg type instability in these models, as will be shown explicitly later in certain directions in field space. Thus the $\alpha_{v 1}^{*}$ perturbative fixed point is classically viable and becomes increasingly flat in the Veneziano limit, and moreover in the absence of relevant operators the flow never leaves the critical curve.

Having identified all the critical coupling values and the scaling dimensions it is possible to parametrize the gauge coupling and hence the entire flow along the critical curve for any values of the renormalization time $t=\ln \mu / \mu_{0}$ by $[3,60]$

$$
\begin{aligned}
& \alpha_{g}(t)=\alpha_{g}^{*}(1+W[t])^{-1}, \\
& \alpha_{y}(t)=\frac{6}{13} \alpha_{g}(t), \\
& \alpha_{h}(t)=3 \frac{\sqrt{23}-1}{26} \alpha_{g}(t), \\
& \alpha_{v}(t)=\frac{3 \sqrt{20+6 \sqrt{23}}-6 \sqrt{23}}{26} \alpha_{g}(t),
\end{aligned}
$$

where $W=\frac{\alpha_{g}^{*}}{\alpha_{g}}-1$ is the Lambert $W$-function (a.k.a. the product $\log$ defined by $W(z) e^{W(z)}=z$ ). We refer to [3] for the explicit relation between $z$ and the RG time $t$. Perturbation theory is valid for all values of $t$ as long as $\epsilon$ is small. We refer to [3] for further details.

Since we can access all scales through this set of solutions, the initial gauge coupling $\alpha_{g}(0)=\alpha_{g}\left(\mu_{0}\right)$ is the only free parameter distinguishing different physical systems that flow from the UV fixed point, and must be set by hand in accord with the measurement of the coupling at some scale. However, as mentioned above one can simply use the gauge coupling itself to parametrize the flow along the critical curve linking the UV interacting fixed point to the IR noninteracting one (also known as the separatrix): it is a monotonically increasing function of $\mu$.

\section{SYMMETRY BREAKING}

What happens when we add a classically relevant operator to such a system, in particular of course a mass-squared term for the scalar $H$ ? As described in the Introduction, as long as the operator remains relevant at the quantum level we do not expect it to affect the UV fixed point, and its status will therefore be equivalent to that of chiral symmetry breaking mass-terms in QCD, in the sense that it is a parameter which is set at the initial RG scale by physical measurement. There is no question of uncontrolled UV sensitivity because we know that the theory is exactly conformal precisely at the UV fixed point (this is of course the central assumption which unlike the $\mathrm{CW}$ mechanism is now motivated by a genuine symmetry). On the other hand being a relevant operator it will divert the flow away from the IR fixed point. In the current context this flow is precisely the seed for radiative symmetry breaking.

\section{A. A simple example}

There are a number of different relevant operators that one might consider adding to the theory that can contribute to symmetry breaking. They are distinguished by whether or not they explicitly break the $S U\left(N_{F}\right)_{L} \times S U\left(N_{F}\right)_{R}$ flavor symmetry of the theory. To be concrete we will first consider the mass term, 


$$
V \supset \frac{m_{\phi}^{2}}{4 N_{F}}\left(\operatorname{Tr}\left(H+H^{\dagger}\right)\right)^{2},
$$

which explicitly breaks the flavor symmetry to the diagonal, $U\left(N_{F}\right)_{L} \times U\left(N_{F}\right)_{R} \rightarrow S U\left(N_{F}\right)_{\text {diag }}$ and picks out just the scalar component of the trace.

Generally, the RG flow will be on a critical surface whose dimensionality is given by the number of relevant operators (plus one), but if this flavor breaking operator is the dominant one, the flow and stability may be analyzed in terms of the corresponding normalized Higgs along its direction,

$$
H=\frac{\phi}{\sqrt{2 N_{F}}} \mathbb{1}_{N_{F} \times N_{F}},
$$

where $\phi$ is real. We will for the moment restrict our attention to only this direction in field space and assume that a negative $m_{\phi}^{2}$ will ultimately be responsible for symmetry breaking - in the next subsection we will focus on the main point of the paper, which is that a positive $m_{\phi}^{2}$ operator radiatively causes instability in other directions.

First let us deal with the quartic part of the classical potential of the theory, which along the $\phi$ direction reads

$$
V_{\text {class }}^{(4)}=\frac{4 \pi^{2}}{N_{F}^{2}}\left(\alpha_{h}+\alpha_{v}\right) \phi^{4} .
$$

Hence we define the effective quartic coupling,

$$
\lambda=32 \pi^{2} \frac{3}{N_{F}^{2}}\left(\alpha_{h}+\alpha_{v}\right) .
$$

It is also useful to define

$$
\kappa=32 \pi^{2} \frac{1}{N_{F}^{2}}\left(3 \alpha_{h}+\alpha_{v}\right) .
$$

In the absence of $m_{\phi}^{2}$ the potential is stable at tree-level, and one can also confirm the one-loop stability [3]. This essentially rules out the $\mathrm{CW}$ form of radiative breaking, because it is not possible perturbatively to take these theories to a limit in which the crucial $M(\phi)^{4} \log M(\phi)^{2}$ terms are dominant. Indeed using the results of the Appendix, the entire one-loop potential along the $\phi$ direction is

$$
\begin{aligned}
V= & \frac{\lambda}{4 !} \phi^{4}+\frac{m_{\phi}^{2}}{2} \phi^{2}+\frac{1}{64 \pi^{2}}\left(m_{\phi}^{2}+\frac{\lambda}{2} \phi^{2}\right)^{2}\left(\log \frac{m_{\phi}^{2}+\frac{\lambda}{2} \phi^{2}}{\mu^{2}}-\frac{3}{2}\right)-\frac{(4 \pi)^{2}}{4 N_{F} N_{C}} \alpha_{y}^{2} \phi^{4}\left(\log \frac{(4 \pi)^{2} \alpha_{y} \phi^{2}}{\sqrt{N_{F} N_{C}} \mu^{2}}-\frac{3}{2}\right) \\
& +\frac{\left(N_{F}^{2}-1\right)}{64 \pi^{2}}\left(\frac{\kappa}{2} \phi^{2}\right)^{2}\left(\log \frac{\frac{\kappa}{2} \phi^{2}}{\mu^{2}}-\frac{3}{2}\right)+\frac{N_{F}^{2}}{64 \pi^{2}}\left(\frac{\lambda}{6} \phi^{2}\right)^{2}\left(\log \frac{\frac{\lambda}{6} \phi^{2}}{\mu^{2}}-\frac{3}{2}\right) .
\end{aligned}
$$

The crucial aspect of this expression is that the last line, which contains the contributions from all the orthogonal Higgs scalars and pseudoscalars that get a mass, are according to Eqs. (11) and (12), suppressed by order $\alpha_{v}$ and $\alpha_{h}$ with respect to the leading term, despite the factor of $N_{F}^{2}$. From one point of view this is of course desirable since it ensures that the theory remains perturbative, but it also means that these terms are not able to play off against the tree-level term in order to create a minimum (in contrast with the original $\mathrm{CW}$ mechanism which without the constraint of having to be on a renormalizable trajectory could freely set $\lambda \sim \alpha_{e}^{2}$ ). It would of course be interesting to find theories where one could (by varying a parameter such as $m_{\phi}^{2}$ ) go continuously to $\mathrm{CW}$ radiative symmetry breaking.

As promised therefore, symmetry breaking, if it occurs at all, must be driven by the mass-squared. Its evolution may be treated in the same way as for any other coupling in a perturbative theory. It is useful for our later treatment of more complicated flavor structure, to have the relevant expressions to hand of the various contributions to the RG flow. For this reason (and to be careful about signs and establish conventions) let us summarize the general framework for a theory of scalars $\phi$ with generic $\phi^{n}$ couplings as

$$
\lambda^{(n)}=\frac{\partial^{n} V}{\partial \phi^{n}},
$$

where of course for the mass-squared we will take $n=2$, so at the risk of confusion $\lambda^{(2)} \equiv m_{\phi}^{2}$. The main equation to solve is the Callan-Symanzik equation for the $n$-point Green's function,

$$
\left(-\frac{\partial}{\partial t}+\bar{\beta} \frac{\partial}{\partial \lambda^{(n)}}-n \bar{\gamma}\right) \lambda_{\text {eff }}^{(n)}=0
$$

where $t=\log \left(\phi / \mu_{0}\right)$, corresponding to invariance under changes in the cutoff $\mu_{0}$, of the coupling $\lambda_{\text {eff }}^{(n)}\left(\phi / \mu_{0}\right)$ that one calculates directly in the effective field theory.

The bars indicate division by $1+\gamma$ : as we will work to one-loop for the evolution of the mass-squareds, they will 
ultimately be dropped. For $n=2$ this gives the anomalous dimension as

$$
\bar{\gamma}=-\frac{1}{2} \frac{\partial \log Z}{\partial t}
$$

where the renormalized fields scale as $\phi \rightarrow \sqrt{Z(t)} \phi$, hence $Z=\exp \left(-2 \int \bar{\gamma} d t\right)$.

In order to solve (15) we identify $\bar{\beta}$ as the $t$-derivative of a running coupling $\lambda(t)$ which must be found by solving

$$
\bar{\beta}=\frac{d \lambda^{(n)}(t)}{d t}=\frac{\partial \lambda_{\mathrm{eff}}^{(n)}}{\partial t}+n \bar{\gamma} \lambda^{(n)},
$$

with the functional form of the right-hand side (RHS) being determined by perturbation theory and Eq. (16). The solution for $\lambda_{\mathrm{eff}}^{(n)}$ is then given in terms of this coupling, by

$$
\lambda_{\text {eff }}^{(n)}=\lambda^{(n)}(t) Z^{n / 2}
$$

In SUSY for example the $t$-derivative of $\lambda_{\text {eff }}^{(n)}$ is zero to all orders due to the non-renormalization theorem, and Eq. (18) simply says that $\lambda^{(n)}(t) \propto Z^{-n / 2}$ : the renormalization of any coupling including masses is multiplicative (thereby solving the hierarchy problem) since it comes entirely from absorbing wave-function renormalization. On the other hand in pure $\lambda \phi^{4}$ theory one has $\gamma=0$ at one-loop and the renormalization of $\lambda$ is dominated by the effective potential.

In the present context we require the anomalous dimension of $H$ to one-loop: it will be denoted by $\gamma$ and is simply [61]

$$
\gamma=\alpha_{y} .
$$

In addition to the field renormalization piece, there is a contribution to the running from the cross-term in the oneloop potential, of the form

$$
V \supset \frac{m_{\phi}^{2}}{2} \phi^{2}\left(1+\frac{\lambda t}{16 \pi^{2}}\right)
$$

where $\lambda \equiv \lambda^{(4)}$ is the quartic coupling. (When we come to discuss radiatively induced breaking later on, this will be the crucial contribution.) As $m_{\phi}^{2}$ is the only coupling with classical dimension, there can be no other contributions to the mass-squared terms at one-loop, as is indeed apparent from Eq. (13). Thus to one-loop (and dropping the bars)

$$
\beta_{m_{\phi}^{2}}=m_{\phi}^{2}\left(\frac{\lambda}{16 \pi^{2}}+2 \gamma\right)
$$

and inserting Eq. (11) gives

$$
\frac{1}{m_{\phi}^{2}} \beta_{m_{\phi}^{2}}=2 \alpha_{y}+\frac{6}{N_{F}^{2}}\left(\alpha_{v}+\alpha_{h}\right) \text {. }
$$

One can conclude that in the Veneziano limit the masssquared renormalization is dominated by the anomalous dimension of the fields and the individual cross-terms die away as $1 / N_{F}^{2}$. Moreover the beta function is always positive indicating that the operator grows (in absolute terms) in the UV but of course always remains relevant. ${ }^{1}$

Substituting the solutions in Eq. (7) we obtain

$$
\frac{1}{m_{\phi}^{2}} \beta_{m_{\phi}^{2}}=f \alpha_{g}
$$

where

$$
f=\frac{12}{13}\left[1+\frac{3}{4 N_{F}^{2}}(\sqrt{20+6 \sqrt{23}}-1-\sqrt{23})\right] .
$$

In the Veneziano limit we find $f \approx 0.92$, with the masssquared growing in the UV as

$$
m_{\phi}^{2} \stackrel{\mathrm{UV}}{\longrightarrow} m_{\phi}(0)^{2}\left(\frac{\mu}{\mu_{0}}\right)^{f \alpha_{g}^{*}} .
$$

Of course the reason this does not disrupt the fixed point is that for parametrically small $\alpha_{g}^{*} \sim \epsilon$ the $m_{\phi}^{2}$ coupling grows much more slowly than $\mu^{2}$ itself. On the other hand the physical mass shrinks in the IR since $\alpha_{g}(t) \rightarrow 0$ there. Indeed integrating Eq. (23) gives the solution

$$
\begin{aligned}
m_{\phi}^{2}(t) & =m_{\phi}^{2}(0) \exp \left[f \int_{0}^{t} \alpha_{g} d t\right] \\
& =m_{\phi}^{2}(0) \omega^{-\frac{3 f}{4 \varepsilon}},
\end{aligned}
$$

where

$$
\omega=\frac{\alpha_{g}^{*} / \alpha_{g}(t)-1}{\alpha_{g}^{*} / \alpha_{g}(0)-1}
$$

Note that the inverse power of $\epsilon$ in (26) does not signal a divergence as $\epsilon$, in fact the opposite is the case, and smaller $\epsilon$ means better perturbativity. Indeed

$$
\log \omega \approx-\frac{4}{3} \epsilon \alpha_{g}^{*} t \sim-\epsilon^{2} t,
$$

so that by the above $m_{\phi}^{2}(t) \sim m_{\phi}^{2}(0) e^{f \alpha_{g}^{*} t}$, and the flow gets smoother as $\epsilon \rightarrow 0$ as expected. This is shown explicitly in Fig. 2.

\footnotetext{
${ }^{1}$ in the technical sense, and hence not relevant in the colloquial sense.
} 


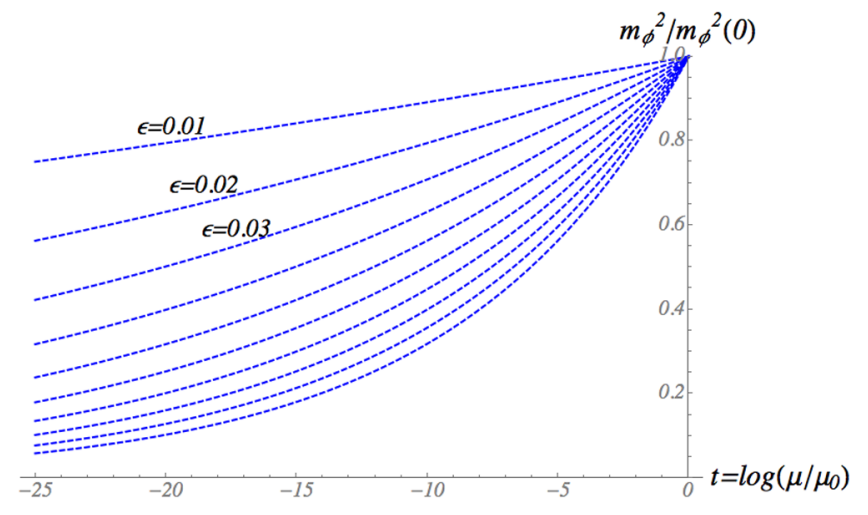

FIG. 2. The behavior of the mass-squared for decreasing $\epsilon$.

We arrive at a purely perturbative description of the evolution of the mass-squared:

$$
m_{\phi}^{2}(t)=m_{*}^{2}\left(\frac{\alpha_{g}^{*}}{\alpha_{g}}-1\right)^{-\frac{3 f}{4 \varepsilon}} \stackrel{\mathrm{IR}}{\longrightarrow} m_{*}^{2}\left(\frac{\alpha_{g}}{\alpha_{g}^{*}}\right)^{\frac{3 f}{4 \varepsilon}},
$$

where the invariant mass-squared parameter is

$$
m_{*}^{2}=m_{\phi}^{2}(0)\left(\alpha_{g}^{*} / \alpha_{g}(0)-1\right)^{\frac{3 f}{4}} .
$$

Note that $m_{*}^{2}$ is independent of the arbitrary energy scale $\mu_{0}$ corresponding to $t=0$ at which the flow started. Therefore each $m_{*}^{2}$ parameter defines a unique trajectory for $m_{\phi}^{2}(t)$, and the totality of possible flows defines a two-dimensional critical surface in $\left(g, y, u, v, m_{\phi}^{2}\right)$-space. The importance of Eq. (29) is that (in accord with the whole philosophy of the renormalisation group) one may now dispense with $\mu_{0}$ and describe the flow entirely in terms of the RG invariants $m_{*}$, $\alpha_{g}^{*}$, and the running coupling $\alpha_{g}(t)$. As was the case for the classically dimensionless couplings, its RG flow is faster by a factor of $1 / \epsilon$ than that of the gauge coupling. Moreover this expression makes transparent the multiplicative nature of the mass renormalization, with the conclusion that in order to have spontaneous symmetry breaking along this direction the parameter $m_{*}^{2}$ has to be negative, implying that $m_{\phi}^{2}$ is negative for all RG scales.

One should of course stop the running around the scale of the Higgs mass which fixes the relevant values of $\alpha_{g}(t)$ and $m_{*}$ for the desired masses and gauge coupling, both of which would in principle be determined by measurement. Note that $\lambda$ also keeps running until the scale of spontaneous symmetry breaking. Therefore the minimum of the tree-level improved potential gives a VEV determined as $\lambda\langle\phi(t)\rangle^{2}=-6 m_{\phi}^{2}(t)$ evaluated with the $t$ parameter corresponding to the value of $\left|m_{\phi}\right|$ itself, which in practice means simply using the appropriate value of $\alpha_{g}$ measured at the scale of the physical Higgs mass.
As one would expect, the spectrum, including that of the quarks, scales as the invariant $m_{*}$ and is otherwise a function only of $\alpha_{g}$ :

$$
\begin{aligned}
m_{\text {higgs }}^{2} /\left|m_{*}^{2}\right| & =2\left(\frac{\alpha_{g}^{*}}{\alpha_{g}}-1\right)^{-\frac{3 f}{4 \epsilon}} \\
m_{Q}^{2} /\left|m_{*}^{2}\right| & =\frac{2 N_{F} / N_{C}}{\sqrt{20+6 \sqrt{63}}-(1+\sqrt{23})}\left(\frac{\alpha_{g}^{*}}{\alpha_{g}}-1\right)^{-\frac{3 f}{4 \epsilon}},
\end{aligned}
$$

where the first of these is simply the usual $m_{\text {higgs }}^{2}=$ $2\left|m_{\phi}^{2}\right|$ relation one has for the Higgs mass of the standard model. There is no color breaking here because the Higgs is a singlet under color, so the gluons remain massless. However one could imagine also gauging the flavor in which case the flavor gauge boson masses would also scale as $m_{*}$, although of course one has to be careful to preserve the asymptotic safety of the whole construct. In summary, $m_{*}$ provides a tunable parameter that, much like the quark masses, encompasses the breaking of both scale invariance and flavor symmetry in the entire flow.

As we hinted above, we are in the above analysis implicitly assuming that a negative $m_{*}^{2}$ leads to instability in the $\phi$ direction alone, and not along any of the other directions. The treatment was also naive in that we have neglected the contribution to the potential of the orthogonal scalars. Their masses are all initially explicitly zero so they do not contribute at leading order, but they will start to contribute loop-suppressed terms proportional to $1 / N_{F}^{2}$ upon resumming the logs. We will see this in a more complete treatment below.

\section{B. Radiative symmetry breaking}

We now begin to extend the discussion to general flavor breaking and first demonstrate that the gaugeYukawa model in Eq. (1) has an in-built mechanism for radiatively induced spontaneous symmetry breaking, analogous to the familiar mechanism of the MSSM. That is, even if a positive parameter such as $m_{\phi}^{2}$ is introduced into the theory at a high renormalization scale, the couplings generically lead to radiative instability in orthogonal directions in field space, and hence to spontaneous breaking of flavor [6]. Moreover we shall see various quite striking similarities with radiative symmetry breaking in the MSSM.

Consider adding to the previous theory a second dimensionful operator that breaks the flavor further as $S U\left(N_{F}\right)_{\text {diag }} \rightarrow S U\left(N_{F} / 2\right)_{\text {diag }} \times S U\left(N_{F} / 2\right)_{\text {diag }}$. We shall discuss the stability for the VEVs of the corresponding (in terms of symmetry breaking) directions in fields space, namely 


$$
H=\frac{\phi}{\sqrt{2 N_{F}}} \mathbb{1}_{N_{F} \times N_{F}}+\frac{h}{\sqrt{2 N_{F}}} \sigma_{1} \otimes \mathbb{1}_{N_{F} / 2 \times N_{F} / 2},
$$

where $\sigma_{1}$ is the usual Pauli matrix. Again the fields $\phi$ and $h$ are the real components of complex fields normalized as for example $\frac{1}{\sqrt{2}}(\phi+i \eta)$. For the one-loop potential one has to of course include the mass-squareds of both the scalar and pseudoscalar fields.

We then add the two mass-squareds for the scalar components into the theory as

$$
V_{\text {class }}^{(2)}=\frac{m_{\phi}^{2}}{2} \phi^{2}+\frac{m_{h}^{2}}{2} h^{2},
$$

with superscript (2) indicating quadratic terms. As in the previous example, the pseudoscalars (and indeed any of the other fields) cannot-initially at least-contribute to the running of these terms, as they do not themselves have an explicit mass-squared and therefore do not have the requisite cross-term in the one-loop potential. On the other hand, as we are about to see for $h$, the converse is not true: even if a mass-squared such as $m_{h}^{2}$ is zero, it gets renormalized by a nonzero $m_{\phi}^{2}$.

Substituting the explicit form of the fields in Eq. (32) into Eq. (1), the quartic terms for $\phi$ and $h$ are

$$
V_{\text {class }}^{(4)}=\frac{\lambda}{4 !}\left(\phi^{4}+h^{4}\right)+\frac{\kappa}{4} \phi^{2} h^{2},
$$

where $\lambda$ and $\kappa$ are as defined in Eqs. (11), (12) and the Appendix. One can alternatively use the expressions in the Appendix to derive the above, noting that, if we call the particular $h$ generator we have chosen here $T^{1}$, then $d_{11 b}=0$ (where $d_{a b c}$ is the totally symmetric $S U\left(N_{F}\right)$ tensor).

To proceed it is useful to define a set of parameters scaled in terms of $\alpha_{g}$ that will encompass the various contributions to the running:

$$
\begin{aligned}
& f_{\gamma} \stackrel{\text { def }}{=} 2 \gamma \frac{1}{\alpha_{g}}=12 / 13, \\
& f_{\lambda} \stackrel{\text { def }}{=} \frac{\lambda}{16 \pi^{2}} \frac{1}{\alpha_{g}}=\frac{9}{13 N_{F}^{2}}[\sqrt{20+6 \sqrt{23}}-1-\sqrt{23}], \\
& f_{\kappa} \stackrel{\text { def }}{=} \frac{\kappa}{16 \pi^{2}} \frac{1}{\alpha_{g}}=\frac{3}{13 N_{F}^{2}}[\sqrt{20+6 \sqrt{23}}-3+\sqrt{23}],
\end{aligned}
$$

where $f=f_{\gamma}+f_{\lambda}$. These ratios (which are all positive) hold along the entire flow (regardless of the presence or otherwise of $m_{\phi}^{2}$ and $m_{h}^{2}$ ). Note that $\gamma$ is dominant in the large $N_{F}$ limit. While the running here is not entirely driven by field renormalization it is as we mentioned earlier certainly dominated by it in the Veneziano limit.
Following the procedure outlined above and applying Eq. (17) for this case we derive two beta functions;

$$
\begin{aligned}
& \beta_{m_{h^{2}}}=\alpha_{g}\left(f m_{h}^{2}+f_{\kappa} m_{\phi}^{2}\right) \\
& \beta_{m_{\phi^{2}}}=\alpha_{g}\left(f m_{\phi}^{2}+f_{\kappa} m_{h}^{2}\right) .
\end{aligned}
$$

These can be diagonalized and solved (using also $\left.\alpha_{g}=\frac{d}{d t} \log w^{-3 / 4 \epsilon}\right)$ to give

$$
\begin{aligned}
& m_{h}^{2}(t)=\frac{w^{-\frac{3 f}{4 \varepsilon}}}{2}\left[m_{h}^{2}(0)\left(w^{\frac{3 f_{\kappa}}{4 \varepsilon}}+w^{-\frac{3 f_{K}}{4 \varepsilon}}\right)-m_{\phi}^{2}(0)\left(w^{\frac{3 f_{K}}{4 \varepsilon}}-w^{-\frac{3 f_{K}}{4 \varepsilon}}\right)\right] \\
& m_{\phi}^{2}(t)=\frac{w^{-\frac{3 f}{4 \varepsilon}}}{2}\left[m_{\phi}^{2}(0)\left(w^{\frac{3 f_{\kappa}}{4 \varepsilon}}+w^{-\frac{3 f_{\kappa}}{4 \varepsilon}}\right)-m_{h}^{2}(0)\left(w^{\frac{3 f_{K}}{4 \varepsilon}}-w^{-\frac{3 f_{K}}{4 \varepsilon}}\right)\right] .
\end{aligned}
$$

Note that the previous result is obtained for $f_{\kappa}=0$ as expected (although we should emphasize that for any given set of operators the $\kappa$ cross-terms and hence $f_{\kappa}$ is completely determined as above).

More importantly however, a large positive value of $m_{\phi}^{2}$ generates a negative $m_{h}^{2}$ and instability in the $h$ direction at low scales (where $w>1$ ) even if all mass-squareds are positive in the $U V$. By the same token a large positive $m_{h}^{2}$ generates a negative $m_{\phi}^{2}$ and instability in the $\phi$ direction.

This phenomenon is purely an effect of the one-loop potential and is precisely what happens in the MSSM, where thanks to supersymmetry, the large (s)top Yukawa generates an effective $\kappa$ driving the soft mass-squared term for the associated Higgs negative [6].

The effect can be made more explicit by noting that in the large $N_{F}$ limit, $\epsilon \sim 1 / N_{F}$ whereas $f_{\kappa} \sim 1 / N_{F}^{2}$. Therefore we can expand as follows;

$m_{h}^{2}(t)=w^{-\frac{3 f}{4 \epsilon}}\left[m_{h}^{2}(0)-m_{\phi}^{2}(0) \frac{3 f_{\kappa}}{4 \epsilon} \log w+\ldots\right]$
$m_{\phi}^{2}(t)=w^{-\frac{3 f}{4 \epsilon}}\left[m_{\phi}^{2}(0)-m_{h}^{2}(0) \frac{3 f_{\kappa}}{4 \epsilon} \log w+\ldots\right]$.

Since the prefactor scales as $\frac{3 f_{\kappa}}{4 \epsilon} \sim 1 / N_{F}$ one must in this simple example ensure that $m_{h}^{2}(0) \lesssim m_{\phi}^{2}(0) / N_{F}$ in order for the symmetry breaking to be driven radiatively by $m_{\phi}^{2}$, so at the moment it appears that the breaking requires an internal hierarchy. This is really a function of the dominance of the field renormalization in the running. Of course a vanishingly small $m_{h}^{2}(0)$ could always be invoked by flavor symmetry arguments, however in the following section we shall present an example that does not require such an assumption. Once the discussion is correctly organized in terms of the $S U\left(N_{F}\right)$ flavor structure the radiative breaking is found to occur when the system does not have any internal hierarchies. 
We should emphasize at this point that the solutions above should be considered to be accurate to one loop and leading log. In principle, and as is about to become clear in the following subsection, all of the $m_{h}^{2}$ pick up a mass-squared in a similar fashion and these then feed back into $m_{\phi}^{2}$ when the logs are resummed during the running. In other words the beta functions for the mass-squareds in all of the orthogonal directions also get contributions from $m_{\phi}^{2}$. It is reasonable to consider only the leading contributions above in particular restricted directions in field space, first because these secondary contributions would be suppressed by more factors of $1 / N_{F}^{2}$, but also because the mass-squareds do not themselves contribute to the fixed point behavior, but simply accumulate contributions perturbatively during the running. Nevertheless we now proceed to improve on the situation with a proper treatment of the flavor structure.

\section{General solutions and the role of flavor}

We conclude from Eq. (37) that adding a large positive mass-squared operator in the UV could generically lead to a further spontaneous radiative breaking of flavor symmetry in a multitude of orthogonal directions. However the value of $m_{h}^{2}(0)$ had to be vanishingly small in the Veneziano limit.

In this subsection we show that in fact the phenomenon occurs generically once one takes into account the large (order $N_{F}^{2}$ ) number of orthogonal degrees of freedom in the Higgs. Indeed as mentioned, there was nothing particularly special about the direction $h$ in the above analysis, compared to any of the other flavor breaking directions that we could have chosen. Therefore in order to identify the correct vacuum one should in principle consider the entire complement of Higgses in the theory.

Let us therefore define the general direction in terms of the generators of flavor (replacing the previous $\phi$ and $\eta$ with $h_{0}$ and $p_{0}$ for convenience),

$$
H=\frac{\left(h_{0}+i p_{0}\right)}{\sqrt{2 N_{F}}} \mathbb{1}_{N_{F} \times N_{F}}+\left(h_{a}+i p_{a}\right) T_{a},
$$

where $T_{a}$ with $a=1 \ldots N_{F}^{2}-1$ labels the adjoint generators of $S U\left(N_{F}\right)_{\text {diag }}$ and by convention $\operatorname{Tr}\left(T_{a} T_{a}\right)=\frac{1}{2}$. The scalar components in the potential are effectively the Hermitian component of $H$ whereas the pseudoscalars are the antiHermitian component.

What is the influence of a positive $m_{h_{0} h_{0}}^{2}$ operator in the other $h_{a}$ directions? The crucial cross-terms in the potential, $V \supset \kappa_{a} h_{0}^{2} h_{a}^{2}$, arise from the $\operatorname{Tr}\left(H^{\dagger} H H^{\dagger} H\right)$ operator in Eq. (1) and as is clear from Eq. (A9) they are all similar in magnitude, and in fact any generators $T^{a}$ that also have $d_{a a b}=0$ receive degenerate mass-squareds. Therefore if for example $m_{h_{a} h_{a}}^{2}(0)=0$ for all the high scale starting values, then all of these directions receive mass-squareds

$$
m_{h_{a} h_{a}}^{2} \approx-\frac{m_{0}^{2}(0)}{2}\left(w^{\frac{3\left(f_{K}-f\right)}{4 e}}-w^{-\frac{3\left(f_{K}+f\right)}{4 e}}\right) \quad \forall a,
$$

where $f_{\kappa}$ is as before, and where the approximation is that we are neglecting cross-terms between the $h_{a}^{2}$ 's which give contributions that are suppressed by powers of $w$. Nevertheless we can conclude that every flavor breaking scalar orthogonal to $h_{0}$ receives a negative mass-squared.

It is interesting to turn the question around and ask when is there guaranteed to be no instability. From Eq. (37), degenerate values of mass-squareds remain degenerate at all scales. This suggests that for all the possible directions to remain stable requires complete degeneracy, $m_{h_{0} h_{0}}^{2} \equiv m_{0}^{2}=m_{h_{a} h_{a}}^{2} \forall a$, which is satisfied if one adds the only mass-squared operator that breaks no flavor symmetry at all, namely $\operatorname{Tr}\left(H^{\dagger} H\right)$.

Therefore in order to find a genuine solution to the $\mathrm{RG}$ equations that one can legitimately resum, one should begin with the RG equations for the most general set of flavor-breaking operators, and seek a deviation from flavor universality that is isomorphic under renormalization: it turns out that a simple suitable structure is generator diagonal and universal except for a flavor deviation in only the trace components; namely

$V_{\text {class }}^{(2)}=m_{0}^{2} \operatorname{Tr}\left(H^{\dagger} H\right)+2 \Delta^{2} \sum_{a} \operatorname{Tr}\left(T_{a} H^{\dagger}\right) \operatorname{Tr}\left(T_{a} H\right)$,

which gives

$$
m_{h_{a} h_{b}}^{2}=m_{p_{a} p_{b}}^{2}=\left(m_{0}^{2}+\Delta^{2}\right) \delta_{a b},
$$

for all the scalar and pseudoscalar $S U\left(N_{F}\right)$ directions, and degenerate trace pseudoscalar and scalar masssquareds, $m_{h_{0} h_{0}}^{2}=m_{p_{0} p_{0}}^{2}=m_{0}^{2}$.

The renormalization of the mass-squared couplings can be determined as before (at the cost of considerably more tedium). The detailed expressions required to build the one-loop potential for the most general case are given in Eq. (A8). Inserting the structure chosen in (41), we find

$$
\begin{aligned}
& \beta_{m_{0}^{2}}=\alpha_{g}\left(f_{m_{0}} m_{0}^{2}+f_{\kappa}^{\Delta} \Delta^{2}\right), \\
& \beta_{\Delta^{2}}=\alpha_{g} f_{\Delta} \Delta^{2},
\end{aligned}
$$

where using the results from Eq. (A21) and inserting the solutions from Eq. (7) we have 


$$
\begin{aligned}
f_{m_{0}} & =\frac{6}{13}\left[\sqrt{20+6 \sqrt{23}}\left(1+\frac{1}{N_{F}^{2}}\right)-\frac{2 \sqrt{23}}{N_{F}^{2}}\right], \\
f_{\kappa}^{\Delta} & =\frac{6}{13}\left(1-\frac{1}{N_{F}^{2}}\right)[\sqrt{20+6 \sqrt{23}}-2], \\
f_{\Delta} & =\frac{6}{13}\left[2+\frac{\sqrt{20+6 \sqrt{23}}-2 \sqrt{23}}{N_{F}^{2}}\right] .
\end{aligned}
$$

Note that $f_{\Delta}$ is dominated by the field renormalization, and that $f_{m_{0}}-f_{\Delta} \approx f_{\kappa}^{\Delta}$ up to corrections of order $1 / N_{F}^{2}$. The crucial aspect of these beta functions is that no degrees of freedom were neglected in their derivation, and this flavor structure remains intact throughout the running. In addition note that $\beta_{\Delta}^{2}$ is zero in the limit of vanishing $\Delta$; as anticipated, totally flavor symmetric mass-squareds do not lead to radiative symmetry breaking as there can be no preferred direction in field space. Finally, in contrast with the simplistic example above, the cross-term in the beta function coefficients does not vanish in the Veneziano limit.

Equation (43) can be solved for $\Delta^{2}$ and the combination

$$
\tilde{m}^{2}=m_{0}^{2}+\nu \Delta^{2}
$$

where we define

$$
\nu=\frac{f_{\kappa}^{\Delta}}{f_{m_{0}}-f_{\Delta}}=1-\frac{1}{N_{F}^{2}} .
$$

Since $f_{\kappa}^{\Delta}>0$ then $f_{m_{0}}>f_{\Delta}$. They have the following solutions;

$$
\tilde{m}^{2}=\tilde{m}^{2}(0) w^{-\frac{3 f m_{0}}{4 \varepsilon}}, \quad \Delta^{2}=\Delta^{2}(0) w^{-\frac{3 f_{\Delta}}{4 \varepsilon}} .
$$

As for the simple case, it is now possible to describe the entire flow in terms of RG invariants; that is defining

$$
\begin{aligned}
& \tilde{m}_{*}^{2}=\tilde{m}^{2}(0)\left(\alpha_{g}^{*} / \alpha_{g}(0)-1\right)^{\frac{3 f m_{0}}{4 \varepsilon}} \\
& \Delta_{*}^{2}=\Delta^{2}(0)\left(\alpha_{g}^{*} / \alpha_{g}(0)-1\right)^{\frac{3 f_{\Delta}}{4 \varepsilon}},
\end{aligned}
$$

one can write

$$
\begin{aligned}
& \tilde{m}^{2}=\tilde{m}_{*}^{2}\left(\frac{\alpha_{g}^{*}}{\alpha_{g}}-1\right)^{-\frac{3 f m_{0}}{4 e}} \\
& \Delta^{2}=\Delta_{*}^{2}\left(\frac{\alpha_{g}^{*}}{\alpha_{g}}-1\right)^{-\frac{3 f_{\Delta}}{4 e}} .
\end{aligned}
$$

With this solution in hand, it is now possible to see how the flavor structure drives radiative symmetry breaking. Consider the case of a slightly positive $\Delta_{*}^{2}$, that is, the $S U\left(N_{F}\right)$ flavor breaking directions are given a slightly larger mass-squared than the trace $h_{0}$ direction. According to Eq. (47) $\tilde{m}^{2}$ shrinks very rapidly in the IR as $w^{-\frac{3 f m_{0}}{4 e}} \rightarrow$ $w^{-2.4 / \epsilon}$ (recalling that $w$ grows in the IR). On the other hand the deviation $\Delta^{2}$ also shrinks, but much more slowly, as $w^{-\frac{3 f_{\Delta}}{4 \epsilon}} \rightarrow w^{-0.7 / \epsilon}$. Because $f_{m_{0}}$ is greater than $f_{\Delta}$, the dominance of $\Delta^{2}$ in the IR is inevitable. Indeed the masssquareds for the different components are

$$
\begin{gathered}
m_{0}^{2}=\tilde{m}_{*}^{2}\left(\frac{\alpha_{g}^{*}}{\alpha_{g}}-1\right)^{-\frac{3 f_{m_{0}}}{4 \varepsilon}}-\Delta_{*}^{2} \nu\left(\frac{\alpha_{g}^{*}}{\alpha_{g}}-1\right)^{-\frac{3 f_{\Delta}}{4 \varepsilon}}, \\
m_{a=1 \ldots N_{F}^{2}-1}^{2}=\tilde{m}_{*}^{2}\left(\frac{\alpha_{g}^{*}}{\alpha_{g}}-1\right)^{-\frac{3 f_{m_{0}}}{4 \varepsilon}}+\Delta_{*}^{2}(1-\nu)\left(\frac{\alpha_{g}^{*}}{\alpha_{g}}-1\right)^{-\frac{3 f_{\Delta}}{4 \varepsilon}},
\end{gathered}
$$

with the $\Delta^{2}$ piece eventually coming to dominate in the IR. Note that since $1-\nu=1 / N_{F}^{2}$, in the large $N_{F}^{2}$ limit light $h_{a}$ directions are collectively driving a much larger negative mass-squared for the single $h_{0}$ direction. (The sum of the mass-squareds is approximately zero). We conclude that a positive $m_{0}^{2}$ is driven entirely negative in the IR if we begin with a preponderance of orthogonal slightly heavier directions in the UV. An example flow is shown in Fig. 3. As is evident from the figure a minimum appears where the deviation $\Delta^{2}$ overcomes the running average mass-squared.

Regardless of the size of flavor breaking, this happens very quickly, and the potential itself develops a minimum at the transmutation scale corresponding to the minimum value of $m_{0}^{2}$; defining

$$
R_{*}=\frac{\Delta_{*}^{2}}{\tilde{m}_{*}^{2}},
$$

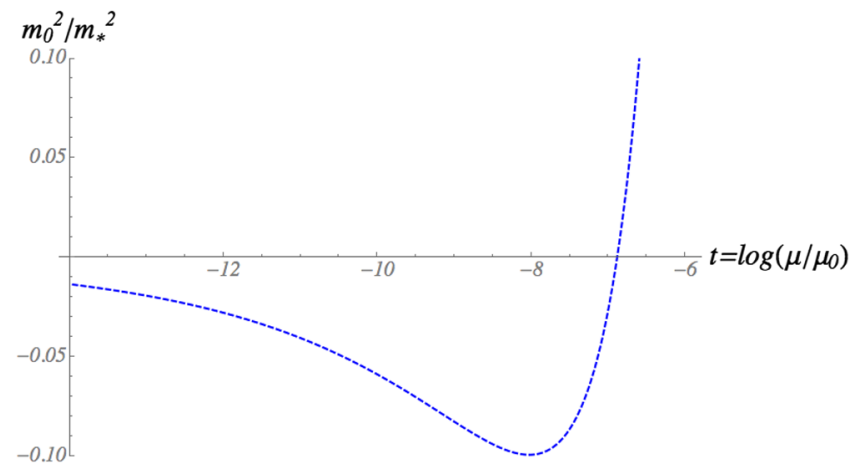

FIG. 3. A trace mass-squared which is smaller than the average by $35 \%$ in the deep UV (i.e. $\Delta_{*}^{2}=0.35$ ) being driven negative radiatively. Note that for reference we take $\mu_{0}$ to be the dynamical scale (i.e., the apparent Landau pole scale) of the low energy theory: it does not indicate the presence of any physical modes at that scale, and the system has no hierarchies. We take $\epsilon=0.1$ in the Veneziano limit $\left(N_{F} \rightarrow \infty\right)$. 
the mass-squared (and hence the potential) forms a minimum at

$$
\begin{aligned}
\frac{\alpha_{g}^{*}}{\alpha_{g, \text { min }}}-1 & \approx\left(\frac{f_{\Delta}}{f_{m_{0}}} \nu R_{*}\right)^{-\frac{4 \varepsilon}{3\left(f_{m_{0}}-f_{\Delta}\right)}}, \\
m_{0, \text { min }}^{2} & \approx-\tilde{m}_{*}^{2} \frac{f_{m_{0}}-f_{\Delta}}{f_{\Delta}}\left(R_{*} \nu \frac{f_{\Delta}}{f_{m_{0}}}\right)^{\frac{f_{m_{0}}}{f_{m_{0}}-f_{\Delta}}} .
\end{aligned}
$$

For the example in Fig. 3, where $R_{*}=0.35$ and $\epsilon=0.1$, the above approximations give $\alpha_{g, \min }=0.47 \alpha_{g}^{*}$ and $m_{0, \min }^{2} \approx-0.1 m_{*}^{2}$, in accord with the numerical plots. Note that for small $\epsilon$ in the Veneziano limit one has

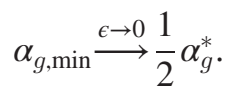

In other words the minimum forms at precisely the scale where the theory is passing from the UV fixed point, and assuming more standard Gaussian IR fixed point behavior. Finally note that if we had chosen negative $\Delta^{2}$ the reversed pattern of breaking would have occurred, with the trace $h_{0}$ direction being the only stable and very heavy direction, with a mass-squared balancing order $N_{F}^{2}$ very small negative mass-squareds for all the orthogonal directions.

\section{CONCLUSIONS}

We have studied the stability properties of the class of perturbative UV fixed point theories introduced in Ref. [1], in the presence of additional scalar mass-squared terms. It is important to realize that such terms, being relevant operators, may take any value in a scenario of asymptotic safety without disrupting the fixed point. As such their status is similar to that of the quark masses in QCD: they are simply set by hand at some scale and are fully controlled and multiplicatively renormalized along the entire RG trajectory. Indeed the value of all the relevant operators everywhere along the flow is completely determined by a set of corresponding RG invariants.

This general picture, in which the trajectories of relevant operators (for example $m_{*}^{2}$ in our case) are determined by a set of tunable RG invariants that defines a particular model, while the marginal operators are all (except for one) determined by a UV fixed point, is a familiar one in the context of the exact renormalization group. However it is certainly novel to be able to treat it perturbatively.

Such a treatment reveals that these theories exhibit an interesting form of calculable radiatively induced symmetry breaking, that is analogous to that in the MSSM [6], in the sense that it is driven by large Yukawa couplings. It was found that a generic set of positive but flavor violating mass-squared terms automatically induce a minimum radiatively, whose depth is determined by the absolute value of the flavor violation. Moreover the minimum inevitably appears at the precise scale where the UV fixed point first loses control over the running, and the theory comes under the more familiar influence of the Gaussian IR fixed point. This is a novel radiative symmetry breaking phenomenon that we believe deserves further study.

There are several issues that require careful treatment in order to embed the standard model in such a scenario. First, there are many gauge bosons that need to be made massive, and moreover once those gauge bosons are made massive they must not then go on to reintroduce a hierarchy problem. Many of these questions are addressed in a companion paper [62]. A rather minimal direct implementation of the radiative symmetry breaking mechanism we have discussed here is to embed the structure into an extended Pati-Salam theory, with a breaking scale somewhat above the weak scale, and with a gauging of the $S U(2)_{L} \times S U(2)_{R}$ subgroup of the flavor symmetry. We should add that in principle the explicit breaking of global flavor symmetries in the scalar mass-squareds is multiplicatively renormalized, so that it may also be possible to realize the Higgs as the Goldstone mode of an approximate global flavor symmetry in such a scenario.

\section{ACKNOWLEDGMENTS}

S. A. A. would like to acknowledge the support of the STFC through the IPPP research grant.

\section{APPENDIX: RESULTS FOR POTENTIAL}

We collect the results required for the computation of the beta functions. The fields contributing to the one-loop potential are decomposed into real and pseudoscalar flavor breaking directions as

$$
H=\frac{h_{0}+i p_{0}}{\sqrt{2 N_{F}}}+\left(h_{a}+i p_{a}\right) T^{a} .
$$

For convenience we use $h_{A} \equiv\left\{h_{0}, h_{1 \ldots N^{2}-1}\right\}$ with

$$
T^{0}=\frac{1}{\sqrt{2 N_{F}}}
$$

and with small letters running as $a=1 \ldots N_{F}^{2}-1$ and capitals as $A=1 \ldots N_{F}^{2}-1$. In principle to determine the correct vacuum one should consider the beta functions of the most general mass-squared terms, are,

$V_{\text {class }}^{(2)}=\frac{1}{2} m_{h_{A} h_{B}}^{2} h_{A} h_{B}+\frac{1}{2} m_{p_{A} p_{B}}^{2} p_{A} p_{B}+m_{p_{A} h_{B}}^{2} p_{A} h_{B}$.

Therefore we examine the potential in the background of all the scalars $h_{A}$ and pseudoscalars $p_{A}$. Using the identity $T_{a} T_{b}=\frac{1}{2}\left(\frac{\delta_{a b}}{N_{F}}+\left(d_{a b k}+i f_{a b k}\right) T^{k}\right)$, the relevant pieces are extracted from the tree-level potential which is 


$$
\begin{aligned}
V_{\text {class }}= & \frac{1}{2} m_{h_{A} h_{B}}^{2} h_{A} h_{B}+\frac{1}{2} m_{p_{A} p_{B}}^{2} p_{A} p_{B}+m_{p_{A} h_{B}}^{2} p_{A} h_{B}+\frac{\left(v+u / N_{F}\right)}{4}\left(h_{A}^{2}+p_{A}^{2}\right)^{2} \\
& +\frac{u}{8}\left(\left(h_{a} h_{b}+p_{a} p_{b}\right) d_{a b k}+2 p_{a} h_{b} f_{a b k}+\frac{4\left(h_{0} h_{k}+p_{0} p_{k}\right)}{\sqrt{2 N_{F}}}\right)\left(\left(h_{c} h_{d}+p_{c} p_{d}\right) d_{c d k}+2 p_{c} h_{d} f_{c d k}+\frac{4\left(h_{0} h_{k}+p_{0} p_{k}\right)}{\sqrt{2 N_{F}}}\right)
\end{aligned}
$$

where $d_{a b c}$ and $f_{a b c}$ are the usual totally symmetric tensor and antisymmetric structure constants respectively of $S U\left(N_{F}\right)$. Note that repeated indices are summed.

The $u$-terms can be significantly simplified by defining

$$
\begin{gathered}
\hat{d}_{A B C}= \begin{cases}d_{A B C} & A, B, C \neq 0 \\
\sqrt{\frac{2}{N_{F}}} \delta_{B C} & A=0 .\end{cases} \\
\hat{f}_{A B C}= \begin{cases}f_{A B C} & A, B, C \neq 0 \\
0 & A \text { or } B \text { or } C=0 .\end{cases}
\end{gathered}
$$

The full tree-level potential then becomes

$$
\begin{aligned}
V_{\text {class }}= & \frac{1}{2} m_{h_{A} h_{B}}^{2} h_{A} h_{B}+\frac{1}{2} m_{p_{A} p_{B}}^{2} p_{A} p_{B}+m_{p_{A} h_{B}}^{2} p_{A} h_{B} \\
& +\frac{v}{4}\left(h_{A}^{2}+p_{A}^{2}\right)^{2}+\frac{u}{8}\left(\left(h_{A} h_{B}+p_{A} p_{B}\right) \hat{d}_{A B K}\right. \\
& \left.+2 p_{A} h_{B} \hat{f}_{A B K}\right)\left(\left(h_{C} h_{D}+p_{C} p_{D}\right) \hat{d}_{C D K}\right. \\
& \left.+2 p_{C} h_{D} \hat{f}_{C D K}\right),
\end{aligned}
$$

The field dependent mass-squareds derived from Eq. (A7) are

$$
\begin{aligned}
M_{h_{A} h_{B}}^{2}= & m_{h_{A} h_{B}}^{2}+v\left(\delta_{A B}\left(h_{C}^{2}+p_{C}^{2}\right)+2 h_{A} h_{B}\right) \\
& +\frac{u}{2} \hat{d}_{A B K}\left(\left(h_{C} h_{D}+p_{C} p_{D}\right) \hat{d}_{C D K}+2 p_{C} h_{D} \hat{f}_{C D K}\right) \\
& +u\left(h_{C} \hat{d}_{A C K}-p_{C} \hat{f}_{A C K}\right)\left(h_{D} \hat{d}_{B D K}-p_{D} \hat{f}_{B D K}\right), \\
M_{p_{A} p_{B}}^{2}= & m_{p_{A} p_{B}}^{2}+v\left(\delta_{A B}\left(h_{C}^{2}+p_{C}^{2}\right)+2 p_{A} p_{B}\right) \\
& +\frac{u}{2} \hat{d}_{A B K}\left(\left(h_{C} h_{D}+p_{C} p_{D}\right) \hat{d}_{C D K}+2 p_{C} h_{D} \hat{f}_{C D K}\right) \\
& +u\left(p_{C} \hat{d}_{A C K}+h_{C} \hat{f}_{A C K}\right)\left(p_{D} \hat{d}_{B D K}+h_{D} \hat{f}_{B D K}\right), \\
M_{p_{A} h_{B}}^{2}= & m_{p_{A} h_{B}}^{2}+2 v p_{A} h_{B} \\
& +\frac{u}{2} \hat{f}_{A B K}\left(\left(h_{C} h_{D}+p_{C} p_{D}\right) \hat{d}_{C D K}+2 p_{C} h_{D} \hat{f}_{C D K}\right) \\
& +u\left(p_{C} \hat{d}_{A C K}+h_{C} \hat{f}_{A C K}\right)\left(h_{D} \hat{d}_{B D K}-p_{D} \hat{f}_{B D K}\right) .
\end{aligned}
$$

Note that when it comes to the renormalization of the masssquareds, only those terms with a direct mass-squared can contribute, although of course all terms contribute to the quartic coupling renormalization in the usual way regardless of the flavor breaking.

It is worth highlighting the generator independence of the above relations: in a background of only real scalars, the terms for $M_{h_{a} h_{a}}^{2}$ and $M_{h_{0} h_{0}}^{2}$ can be rewritten

$M_{h_{a} h_{a}}^{2}=m_{a}^{2}+\frac{\lambda}{2} h_{a}^{2}+\frac{\kappa}{2} h_{C \neq a}^{2}+$ cross-terms involving $d_{a b c}$,

$M_{h_{0} h_{0}}^{2}=m_{h_{0}}^{2}+\frac{\lambda}{2} h_{0}^{2}+\frac{\kappa}{2} h_{a}^{2}$,

where as in the text, the coefficients are

$$
\begin{aligned}
& \lambda / 2=3\left(\frac{u}{N_{F}}+v\right)=\frac{3(4 \pi)^{2}}{N_{F}^{2}}\left(\alpha_{h}+\alpha_{v}\right), \\
& \kappa / 2=\left(3 \frac{u}{N_{F}}+v\right)=\frac{(4 \pi)^{2}}{N_{F}^{2}}\left(3 \alpha_{h}+\alpha_{v}\right) .
\end{aligned}
$$

\section{The degenerate example}

Now let us specialize to the specific generator-diagonal structure considered in the text,

$V_{\text {class }}^{(2)}=m_{0}^{2} \operatorname{Tr}\left(H H^{\dagger}\right)+2 \Delta^{2} \delta_{a b} \operatorname{Tr}\left(H T^{a}\right) \operatorname{Tr}\left(H^{\dagger} T^{b}\right)$.

We will derive $f_{m_{0}^{2}}$ and $f_{\Delta^{2}}$ in the beta function for $m_{0}^{2}$ and $\Delta^{2}$, defined as

$\beta_{m_{0}^{2}}=\alpha_{g}\left(f_{m_{0}} m_{0}^{2}+f_{\kappa}^{\Delta} \Delta^{2}\right), \quad \beta_{\Delta^{2}}=\alpha_{g} f_{\Delta} \Delta^{2}$.

Note that we do not expect to find a term proportional to $m_{0}^{2}$ in $\beta_{\Delta^{2}}$ because the completely flavor-symmetric system should be stable against radiative corrections to $\Delta$, so its beta function should vanish when $\Delta=0$. Note also that $f_{m_{0}} \alpha_{g} \equiv \gamma_{\hat{S}}$ can be identified as the anomalous dimensions of the operator $\hat{S}=\operatorname{Tr}\left(H H^{\dagger}\right)$. This will give us a useful cross-check with the results of Ref. [61].

Inserting Eq. (A11) into Eq. (A8), we find the following cross-terms contributing to the mass-squareds in the oneloop potential; 


$$
\begin{aligned}
\partial_{t} V^{\left(m_{h_{a} h_{a}}^{2}\right)}= & \frac{\left(m_{0}^{2}+\Delta^{2}\right)}{16 \pi^{2}} \sum_{a}\left(v\left[2 h_{a}^{2}+\sum_{C}\left(h_{C}^{2}+p_{C}^{2}\right)\right]\right. \\
& \left.+\frac{u}{N_{F}}\left[\sum_{C}\left(h_{C}^{2}+p_{C}^{2}\right)+2 h_{a}^{2}+2 h_{0}^{2}\right]\right) \\
& +\frac{\left(m_{0}^{2}+\Delta^{2}\right)}{16 \pi^{2}} \sum_{a} u\left[h_{c} h_{d} d_{a c k} d_{a d k}\right. \\
& \left.+p_{c} p_{d} f_{a c k} f_{a d k}\right], \\
\partial_{t} V^{\left(m_{p_{a p} p_{a}}^{2}\right)}= & \frac{\left(m_{0}^{2}+\Delta^{2}\right)}{16 \pi^{2}} \sum_{a}\left(v\left[2 p_{a}^{2}+\sum_{C}\left(h_{C}^{2}+p_{C}^{2}\right)\right]\right. \\
& \left.+\frac{u}{N_{F}}\left[\sum_{C}\left(h_{C}^{2}+p_{C}^{2}\right)+2 p_{a}^{2}+2 p_{0}^{2}\right]\right) \\
& +\frac{\left(m_{0}^{2}+\Delta^{2}\right)}{16 \pi^{2}} \sum_{a} u\left[p_{c} p_{d} d_{a c k} d_{a d k}\right. \\
& \left.+h_{c} h_{d} f_{a c k} f_{a d k}\right], \\
\partial_{t} V^{\left(m_{h_{0} h_{0}}^{2}\right)}= & \frac{m_{0}^{2}}{16 \pi^{2}}\left(v\left[2 h_{0}^{2}+\sum_{C}\left(h_{C}^{2}+p_{C}^{2}\right)\right]\right. \\
& \left.+\frac{u}{N_{F}}\left[\sum_{C}\left(3 h_{C}^{2}+p_{C}^{2}\right)\right]\right), \\
& \left.+\frac{u}{N_{F}}\left[\sum_{C}\left(h_{C}^{2}+3 p_{C}^{2}\right)\right]\right), \\
& \frac{m_{0}^{2}}{16 \pi^{2}}\left(v\left[2 p_{0}^{2}+\sum_{C}\left(h_{C}^{2}+p_{C}^{2}\right)\right]\right.
\end{aligned}
$$

where for example $\partial_{t} V^{\left(m_{h_{a} h_{a}}^{2}\right)}$ denotes the terms coming from cross-products with $m_{h_{a} h_{a}}^{2}$. Using the standard $S U\left(N_{F}\right)$ identities (repeated indiced summed),

$$
\begin{aligned}
d_{a a k} & =0 ; \quad d_{a c k} d_{a d k}=\frac{N_{F}^{2}-4}{N_{F}} \delta_{c d} ; \\
f_{a c k} d_{a d k} & =0 ; \quad f_{a c k} f_{a d k}=N_{F} \delta_{c d},
\end{aligned}
$$

and summing we find,

$$
\begin{aligned}
\partial_{t} V^{\left(m_{h_{a} h_{a}}^{2}\right)}+\partial_{t} V^{\left(m_{\text {papa }}^{2}\right)} & \\
= & \frac{\left(m_{0}^{2}+\Delta^{2}\right)}{16 \pi^{2}}\left[2 v \sum\left(h_{a}^{2}+p_{a}^{2}\right)\right. \\
& \left.+2\left(v+\frac{2 u}{N_{F}}\right)\left(N_{F}^{2}-1\right) \sum\left(h_{C}^{2}+p_{C}^{2}\right)\right],
\end{aligned}
$$

where there are some notable cancellations of the structure constant terms, and

$$
\begin{aligned}
\partial_{t} V^{\left(m_{h_{0} h_{0}}^{2}\right)}+\partial_{t} V^{\left(m_{p_{0} p_{0}}^{2}\right)} & \\
= & \frac{m_{0}^{2}}{16 \pi^{2}}\left(4\left(v+\frac{u}{N_{F}}\right)\left[\sum_{C}\left(h_{C}^{2}+p_{C}^{2}\right)\right]\right. \\
& \left.-2 v \sum_{a}\left(h_{a}^{2}+p_{a}^{2}\right)\right) .
\end{aligned}
$$

In total then, the contributions can be divided into pieces proportional to $\sum_{C}\left(h_{C}^{2}+p_{C}^{2}\right)$ that contribute to $\beta_{m_{0}^{2}}$, and proportional to $\sum_{a}\left(h_{a}^{2}+p_{a}^{2}\right)$ that contribute to the running of $\Delta^{2}$ :

$$
\begin{aligned}
\partial_{t} V= & \frac{m_{0}^{2}}{16 \pi^{2}}\left[2 v\left(N_{F}^{2}+1\right)+4 u N_{F}\right] \sum_{C}\left(h_{C}^{2}+p_{C}^{2}\right) \\
& +\frac{\Delta^{2}}{16 \pi^{2}}\left[2\left(v+\frac{2 u}{N_{F}}\right)\left(N_{F}^{2}-1\right)\right] \sum_{C}\left(h_{C}^{2}+p_{C}^{2}\right) \\
& +\frac{\Delta^{2}}{16 \pi^{2}} 2 v \sum_{a}\left(h_{a}^{2}+p_{a}^{2}\right) .
\end{aligned}
$$

As expected the terms proportional to $m_{0}^{2} \sum_{a}\left(h_{a}^{2}+p_{a}^{2}\right)$ cancel in a nontrivial manner.

The beta function coefficients are found (as in the main body of the text) by reading off (twice) the coefficient of the corresponding term in $\partial_{t} V$, and adding $2 \gamma=2 \alpha_{y}$ to the diagonal pieces for the anomalous dimension of the fields. We find

$$
\begin{aligned}
f_{m_{0}} \alpha_{g} & =2 \alpha_{y}+4 \alpha_{v}\left(1+\frac{1}{N_{F}^{2}}\right)+8 \alpha_{h}, \\
f_{\kappa}^{\Delta} \alpha_{g} & =\left(4 \alpha_{v}+8 \alpha_{h}\right)\left(1-\frac{1}{N_{F}^{2}}\right), \\
f_{\Delta} \alpha_{g} & =2 \alpha_{y}+\frac{4}{N_{F}^{2}} \alpha_{v} .
\end{aligned}
$$

As a check we can confirm that the first of these is the anomalous dimension $\gamma_{\hat{S}}$ of the singlet composite operator, calculated in [61]. For use in the text note that

$$
\nu=\frac{f_{\kappa}^{\Delta}}{f_{m_{0}}-f_{\Delta}}=1-\frac{1}{N_{F}^{2}} .
$$


[1] D. F. Litim and F. Sannino, Asymptotic safety guaranteed, J. High Energy Phys. 12 (2014) 178.

[2] O. Antipin, M. Gillioz, E. Mølgaard, and F. Sannino, The a theorem for Gauge-Yukawa theories beyond Banks-Zaks, Phys. Rev. D 87, 125017 (2013).

[3] D. F. Litim, M. Mojaza, and F. Sannino, Vacuum stability of asymptotically safe gauge-Yukawa theories, J. High Energy Phys. 01 (2016) 081.

[4] B. Bajc and F. Sannino, Asymptotically safe grand unification, J. High Energy Phys. 12 (2016) 141.

[5] G. M. Pelaggi, F. Sannino, A. Strumia, and E. Vigiani, Naturalness of asymptotically safe Higgs, arXiv:1701 .01453 .

[6] L. E. Ibanez and G. G. Ross, $\mathrm{SU}(2)_{L} \times U(1)$ symmetry breaking as a radiative effect of supersymmetry breaking in GUTs, Phys. Lett. 110B, 215 (1982).

[7] A. Salam and J. A. Strathdee, Nonlinear realizations. II. Conformal symmetry, Phys. Rev. 184, 1760 (1969); J. R. Ellis, Aspects of conformal symmetry and chirality, Nucl. Phys. B22, 478 (1970).

[8] S. R. Coleman and E. J. Weinberg, Radiative corrections as the origin of spontaneous symmetry breaking, Phys. Rev. D 7, 1888 (1973).

[9] E. Gildener and S. Weinberg, Symmetry breaking and scalar bosons, Phys. Rev. D 13, 3333 (1976).

[10] W. A. Bardeen, Report No. FERMILAB-CONF-95-391-T.

[11] K. A. Meissner and H. Nicolai, Conformal symmetry and the standard model, Phys. Lett. B 648, 312 (2007).

[12] W.-F. Chang, J. N. Ng, and J. M. S. Wu, Shadow Higgs from a scale-invariant hidden U(1)(s) model, Phys. Rev. D 75, 115016 (2007).

[13] T. Hambye and M.H. G. Tytgat, Electroweak symmetry breaking induced by dark matter, Phys. Lett. B 659, 651 (2008).

[14] W. D. Goldberger, B. Grinstein, and W. Skiba, Distinguishing the Higgs Boson from the Dilaton at the Large Hadron Collider, Phys. Rev. Lett. 100, 111802 (2008).

[15] R. Foot, A. Kobakhidze, K. L. McDonald, and R. R. Volkas, A solution to the hierarchy problem from an almost decoupled hidden sector within a classically scale invariant theory, Phys. Rev. D 77, 035006 (2008).

[16] S. Iso, N. Okada, and Y. Orikasa, Classically conformal $B^{-}$L extended standard model, Phys. Lett. B 676, 81 (2009).

[17] K. A. Meissner and H. Nicolai, Conformal invariance from non-conformal gravity, Phys. Rev. D 80, 086005 (2009).

[18] M. Holthausen, M. Lindner, and M. A. Schmidt, Radiative symmetry breaking of the minimal left-right symmetric model, Phys. Rev. D 82, 055002 (2010).

[19] L. Alexander-Nunneley and A. Pilaftsis, The minimal scale invariant extension of the standard model, J. High Energy Phys. 09 (2010) 021.

[20] T. Hur and P. Ko, Scale Invariant Extension of the Standard Model with Strongly Interacting Hidden Sector, Phys. Rev. Lett. 106, 141802 (2011).

[21] C. Coriano, L. Delle Rose, A. Quintavalle, and M. Serino, Dilaton interactions and the anomalous breaking of scale invariance of the standard model, J. High Energy Phys. 06 (2013) 077.
[22] A. R. Vieira, B. Hiller, M. D. R. Sampaio, and M. C. Nemes, Naturalness and theoretical constraints on the Higgs boson mass, Int. J. Theor. Phys. 52, 3494 (2013).

[23] Z. Chacko and R. K. Mishra, Effective theory of a light dilaton, Phys. Rev. D 87, 115006 (2013).

[24] S. Iso and Y. Orikasa, TeV Scale B-L model with a flat Higgs potential at the Planck scale-in view of the hierarchy problem, Prog. Theor. Exp. Phys. 2013, 023 B08 (2013).

[25] C. Englert, J. Jaeckel, V. V. Khoze, and M. Spannowsky, Emergence of the electroweak scale through the Higgs portal, J. High Energy Phys. 04 (2013) 060.

[26] M. Farina, D. Pappadopulo, and A. Strumia, A modified naturalness principle and its experimental tests, J. High Energy Phys. 08 (2013) 022.

[27] M. Fabbrichesi and S. T. Petcov, Low-scale neutrino seesaw mechanism and scalar dark matter, Eur. Phys. J. C 74, 2774 (2014).

[28] J. Lykken, talk at the MITP Workshop "The First Three Years of the LHC," Mainz, March 18-22, 2013.

[29] E. J. Chun, S. Jung, and H. M. Lee, Radiative generation of the Higgs potential, Phys. Lett. B 725, 158 (2013).

[30] M. Heikinheimo, A. Racioppi, M. Raidal, C. Spethmann, and K. Tuominen, Physical naturalness and dynamical breaking of classical scale invariance, Mod. Phys. Lett. A 29, 1450077 (2014).

[31] I. Oda, Classically scale-invariant B-L model and conformal gravity, Phys. Lett. B 724, 160 (2013).

[32] S. Dubovsky, V. Gorbenko, and M. Mirbabayi, Natural tuning: Towards a proof of concept, J. High Energy Phys. 09 (2013) 045.

[33] T. Hambye and A. Strumia, Dynamical generation of the weak and dark matter scale, Phys. Rev. D 88, 055022 (2013).

[34] V. V. Khoze and G. Ro, Leptogenesis and neutrino oscillations in the classically conformal standard model with the Higgs portal, J. High Energy Phys. 10 (2013) 075.

[35] D. Gorbunov and A. Tokareva, Scale-invariance as the origin of dark radiation?, Phys. Lett. B 739, 50 (2014).

[36] C. D. Carone and R. Ramos, Classical scale-invariance, the electroweak scale and vector dark matter, Phys. Rev. D 88, 055020 (2013).

[37] A. Farzinnia, H. J. He, and J. Ren, Natural electroweak symmetry breaking from scale invariant Higgs mechanism, Phys. Lett. B 727, 141 (2013).

[38] D. Chway, T. H. Jung, H. D. Kim, and R. Dermisek, Radiative Electroweak Symmetry Breaking Model Perturbative All the Way to the Planck Scale, Phys. Rev. Lett. 113, 051801 (2014).

[39] S. Y. Choi, C. Englert, and P. M. Zerwas, Multiple Higgsportal and gauge-kinetic mixings, Eur. Phys. J. C 73, 2643 (2013).

[40] V. V. Khoze, Inflation and dark matter in the Higgs portal of classically scale invariant standard model, J. High Energy Phys. 11 (2013) 215.

[41] C. Tamarit, Running couplings with a vanishing scale anomaly, J. High Energy Phys. 12 (2013) 098.

[42] E. Gabrielli, M. Heikinheimo, K. Kannike, A. Racioppi, M. Raidal, and C. Spethmann, Towards completing the standard model: Vacuum stability, EWSB and dark matter, Phys. Rev. D 89, 015017 (2014). 
[43] R. Foot, A. Kobakhidze, K. L. McDonald, and R. R. Volkas, Poincar protection for a natural electroweak scale, Phys. Rev. D 89, 115018 (2014).

[44] O. Antipin, M. Mojaza, and F. Sannino, Conformal extensions of the standard model with Veltman conditions, Phys. Rev. D 89, 085015 (2014).

[45] T. G. Steele, Z. W. Wang, D. Contreras, and R. B. Mann, Viable Dark Matter via Radiative Symmetry Breaking in a Scalar Singlet Higgs Portal Extension of the Standard Model, Phys. Rev. Lett. 112, 171602 (2014).

[46] M. Hashimoto, S. Iso, and Y. Orikasa, Radiative symmetry breaking at the Fermi scale and flat potential at the Planck scale, Phys. Rev. D 89, 016019 (2014).

[47] M. Holthausen, J. Kubo, K. S. Lim, and M. Lindner, Electroweak and conformal symmetry breaking by a strongly coupled hidden sector, J. High Energy Phys. 12 (2013) 076.

[48] S. Abel and A. Mariotti, Novel Higgs potentials from gauge mediation of exact scale breaking, Phys. Rev. D 89, 125018 (2014).

[49] T. Banks and A. Zaks, On the phase structure of vector-like gauge theories with massless fermions, Nucl. Phys. B196, 189 (1982).

[50] S. Weinberg, Report No. C76-07-23.1 HUTP-76/160, Erice Sub- nucl. Phys., 1, 1976.

[51] S. P. Martin and J. D. Wells, Constraints on ultraviolet stable fixed points in supersymmetric gauge theories, Phys. Rev. D 64, 036010 (2001).

[52] H. Gies, J. Jaeckel, and C. Wetterich, Towards a renormalisable standard model without fundamental Higgs scalar, Phys. Rev. D 69, 105008 (2004).
[53] M. Shaposhnikov and D. Zenhausern, Quantum scale invariance, cosmological constant and hierarchy problem, Phys. Lett. B 671, 162 (2009); Z. Chacko and R. K. Mishra, Effective theory of a light dilaton, Phys. Rev. D 87, 115006 (2013).

[54] H. Gies, S. Rechenberger, and M. M. Scherer, Towards an asymptotic-safety scenario for chiral Yukawa systems, Eur. Phys. J. C 66, 403 (2010).

[55] J. Braun, H. Gies, and D. D. Scherer, Asymptotic safety: A simple example, Phys. Rev. D 83, 085012 (2011).

[56] F. Bazzocchi, M. Fabbrichesi, R. Percacci, A. Tonero, and L. Vecchi, Fermions and Goldstone bosons in an asymptotically safe model, Phys. Lett. B 705, 388 (2011).

[57] C. Wetterich, Where to look for solving the gauge hierarchy problem?, Phys. Lett. B 718, 573 (2012).

[58] H. Gies, S. Rechenberger, M. M. Scherer, and L. Zambelli, An asymptotic safety scenario for gauged chiral HiggsYukawa models, Eur. Phys. J. C 73, 2652 (2013).

[59] G. Marques Tavares, M. Schmaltz, and W. Skiba, Higgs mass naturalness and scale invariance in the UV, Phys. Rev. D 89, 015009 (2014).

[60] D. F. Litim, Renormalisation group and the Planck scale, Phil. Trans. R. Soc. A 369, 2759 (2011).

[61] O. Antipin, E. Mø lgaard, and F. Sannino, Higgs critical exponents and conformal bootstrap in four dimensions, J. High Energy Phys. 06 (2015) 030.

[62] S. Abel and F. Sannino, Framework for an asymptotically safe standard model via dynamical breaking, Phys. Rev. D 96, 055021 (2017). 